\title{
MadGraph/MadEvent v4: from models to events
}

\author{
Rikkert Frederix* \\ Centre for Particle Physics and Phenomenology (CP3) \\ Université Catholique de Louvain \\ Chemin du cycltron 2, B-1348 Louvain-La-Neuve, Belgium \\ E-mail: rikkert.frederix@uclouvain.be
}

The new version of the MadGraph/MadEvent (MG/ME) MC generator is introduced by emphasizing its easy-to-use way of incorporating new models and generating events. As an application, a study of $t \bar{t}$ production in various scenarios beyond the standard model is presented.

CP3-07-21

XI International Workshop on Advanced Computing and Analysis Techniques in Physics Research April 23-27 2007

Amsterdam, the Netherlands

${ }^{*}$ Speaker. 


\section{Introduction to MadGraph/MadEvent}

With the start of the LHC drawing closer the need for efficient and reliable predictions for both Standard Model (SM) as well as Beyond the Standard Model (BSM) physics is becoming more important. MadGraph/MadEvent (MG/ME) [1, 2, 3] is a fully automatic MonteCarlo that can contribute to this endeavor. MadGraph is a tree-level matrix-element generator based on the HELAS library [П]. Upon a user's request, it produces the code for a given process and the information necessary to an efficient integration over the phase space. Calculation of the cross section and event generation is then performed by MadEvent using 'single-diagram-enhanced multichannel integration', which not only gives high unweighting efficiencies, but also the possibility for parallelization of the integration. This makes this technique suitable for running on computer farms or clusters leading to a quick event generation. Parton-level events are written out in the LH format and can be passed to parton-shower and hadronization codes, such as Pythia [5] or Herwig [6].

In designing a code, not only algorithms and technical solutions are important, but also human factors need to be taken into account. Lifting the user from the burden of necessarily knowing the details of a code, can lead to significant gains in working efficiency. The MG/ME approach to these issues is based on the possibility of accessing to event generation from different levels, depending on the user's expertise. The simplest way is to log onto one of our web servers and perform the generation via the web, all the way down to detector level, with just a few mouse clicks. All the relevant information, from event sets to plots is stored in a personal database and can be downloaded at any later time for further analysis.

For the more advanced or demanding users, the code specific to one process, or the full MG/ME platform with all its applications and utilities can be also downloaded. One typical example is when his/hers favorite model for BSM physics is not available on-line. The user can download the (open-source) code for MadGraph/MadEvent and add the model through a specifically designed framework which makes the implementation of new particles and interactions effortless.

In the following I illustrate the MG/ME platform features by discussing how to introduce, test and perform a physics study on the discovery of a new resonance at the LHC.

\subsection{The structure of MG/ME}

In Fig. 1 the flow chart for MadGraph/MadEvent is shown. The generation of events up to detector level proceeds in four steps. The first is the creation of the Feynman diagrams and HELAS amplitudes which is accomplished by MadGraph. In the second step the matrix elements corresponding to the various subprocesses (for hadron collisions) are integrated over the phase space and unweighted parton-level events are generated. Then, if the user wishes, the events can be showered and hadronized using Pythia, and finally PGS [0] can be used as a generic detector simulation to obtain reconstructed objects. Once the (parton, hadron, and reconstructed level) events are generated, they can be analyzed using ExRootAnalysis or MadAnalysis, two especially designed packages for experimentalists and theorists, respectively. All these steps are steered by 'cards' which are simple text files that provide all the necessary information.

The generation chain is independent of the collider type or the physics model. As long as the model is available or has been developed (and tested) in the 'User model' (usrmod) framework 


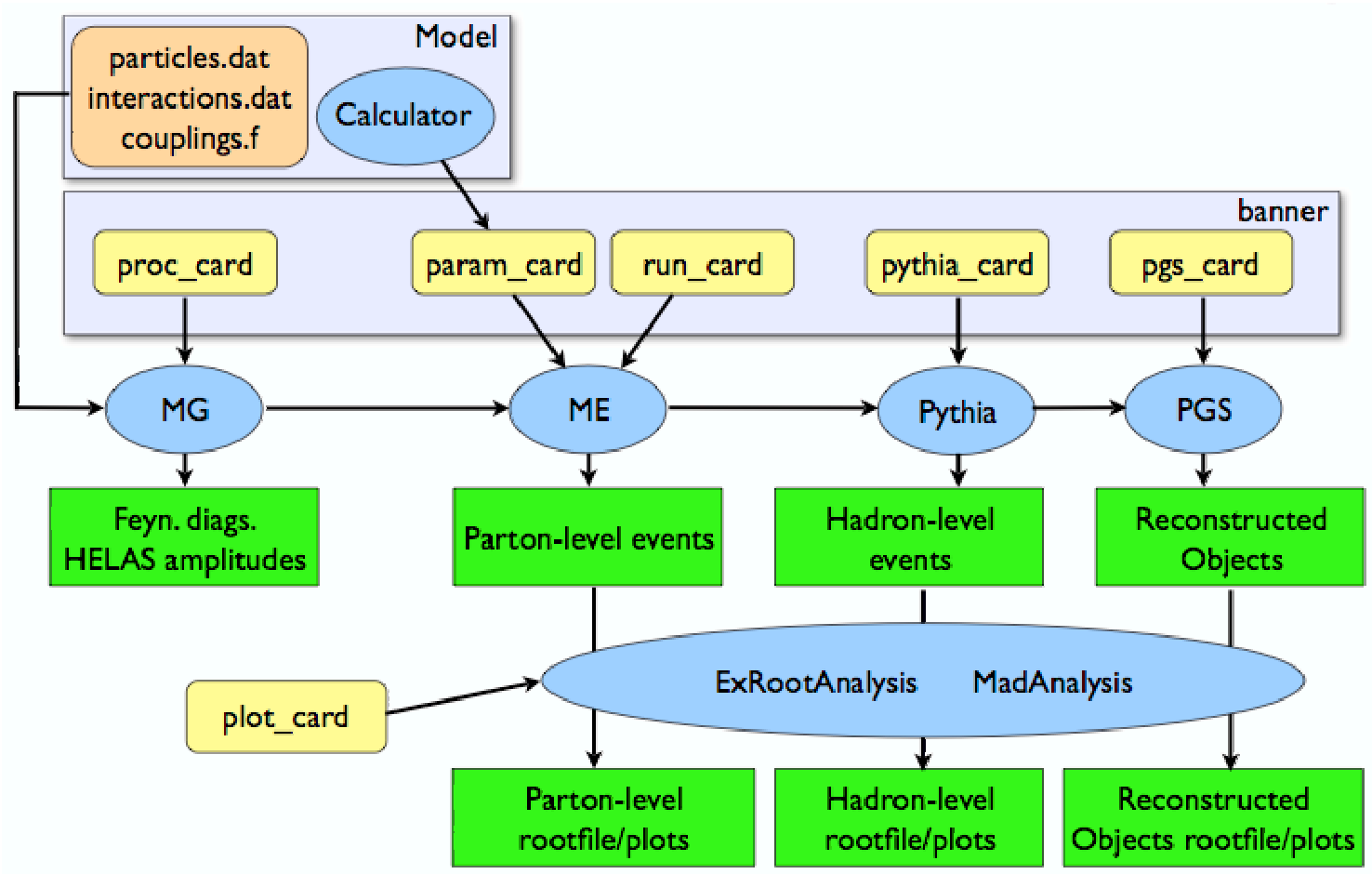

Figure 1: The MadGraph/MadEvent flow chart.

the process can be generated and all the other steps can be taken automatically. At present there are several models implemented and validated in MadGraph:

- the Standard Model (SM), including effective couplings between gluons/photons and Higgs bosons (HEFT);

- the Minimal Super-symmetric Standard Model (MSSM), R-parity conserving and no additional CP violation, but no super-symmetry breaking scheme is assumed [8];

- the Two Higgs doublet Model (2HDM), completely general with all couplings allowed by gauge invariance and electric charge conservation.

Other models, such as Universal Extra Dimensions [9], are currently being developed and tested.

\subsection{User Model}

The User Model is a framework for the implementation of new particles and interactions. It simplifies the coding needed for adding new particles and interactions to modifying/adding a limited number of lines in text files. Within the User Model it is trivial to implement new spin-0 (scalars), spin-1/2 (fermions), spin-1 (vectors), and, with a little bit more work, spin-2 particles too. A rich set of Lorentz (and color structures) for interactions of the new particles are allowed, based on the availability of the corresponding HELAS routines. 


\section{2. $X$ phenomenology}

Let us consider a new model predicting the existence of an heavy state, $X$, whose phenomenology we are interested in, and study the simplest of all possible production mechanism, together with its decay into a pair of leptons and top-anti-top pair, i.e., $p p \rightarrow X \rightarrow \mu^{+} \mu^{-}, t \bar{t}$. The strategy is to look at its effects on the dimuon and $t \bar{t}$ invariant mass spectrum, and possibly use spin correlations between the decay products to determine its angular momentum.

\subsection{Implementation}

As a simple example we show how to implement a new spin-1 particle, a $Z^{\prime}$, within the framework of the user model. This is a typical example; the implementation of other particles is similar. First, we have to add this particle to the list of already available SM particles. In the particle list file (particles.dat), an extra $Z$ appears as

\begin{tabular}{|c|c|c|c|c|c|c|c|c|c|}
\hline$\#$ & Name & anti-Name & Spin & Linetype & Mass & Width & Color & Label & Model \\
\hline \# & $\mathrm{xxxx}$ & $\mathrm{xxxx}$ & SFV & WSDC & str & str & STO & str & PDG code \\
\hline \multicolumn{10}{|c|}{$\cdots$} \\
\hline & MODEL & EXTENSION & & & & & & & \\
\hline & $z p$ & $\mathrm{zp}$ & V & W & ZPMASS & ZPWIDTH & $\mathrm{S}$ & $\mathrm{ZP}$ & 32 \\
\hline
\end{tabular}

where the quantum numbers and the other properties of the $Z^{\prime}$ are given in a simple syntax. In this example we have a $Z^{\prime}(\mathrm{zp})$ of vector type (V), with mass and width ZPMASS and ZPWIDTH. We define it as a color singlet by setting color to $\mathrm{S}$. The line type $\mathrm{W}$ and the label $\mathrm{ZP}$ are used in the Feynman diagrams for cosmetics only.

In the second file that has to be modified, interactions. dat, the possible interactions between the particles are listed. For example, the vertex between the new $Z^{\prime}$ and two top quarks is given by

$$
\begin{aligned}
& \# \text { USRVertex } \\
& t \text { t zP GZPT QED }
\end{aligned}
$$

GZPT is the name for the coupling constant, QED indicates the type of interaction. The coupling of the $Z^{\prime}$ to the other quarks and the leptons may be added in a similar way.

Now, by simply running a script, ConversionScript.pl. a Fortran file, couplings .f, is updated and a parameter card, param_card.dat, generated, so that all the new couplings variables are correctly named and defined. As a last step the user can fill in the numerical values (or the analytic formulas) needed for the couplings. The parameter card is a file in the LHA format [10], which lists all parameters of a model, such as masses, widths and coupling constants. The User Model parameter card is similar to the SM card, but also includes the masses and widths of the new particles. In the cases of width calculations, which is of course model dependent, the user can exploit the power of BRIDGE [11] to calculate the widths of the new particles in the same framework.

By editing couplings. $f$ we can easily assign the strength of the couplings of the $Z$ ' to the top quarks to

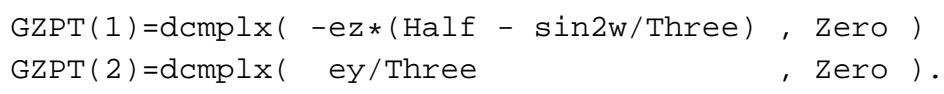

The implementation of spin- 0 and spin- 2 particles is similar. For a more detailed description on the implementation of BSM physics using the User Model refer the README file in the us rmod directory. 


\subsection{Resonances in the dimuon channel}

Processes with leptons (in particular electrons and muons) in the final state are very clean at the LHC, both theoretically and experimentally,. Leptons are relatively easy to detect and trigger on, and their momenta can be measured with a high precision. From the theory side, QED processes have in general small corrections from higher orders. If the coupling of the $X$ resonance to leptons is not too small, the dilepton production channel is the discovery channel for heavy resonances. As an example, in Fig. 目 the dimuon invariant mass is plotted for a $1 \mathrm{TeV} Z^{\prime}$ resonance, with couplings equal to the couplings of the SM $Z$ boson to fermions. The interference effects between the $Z^{\prime}$ and the $Z$ bosons lead to a slightly smaller (larger) cross section for invariant masses below (above) the $Z^{\prime}$ mass as compared to the naïve sum of the contributions from the $Z / \gamma$ and the $Z^{\prime}$ bosons.

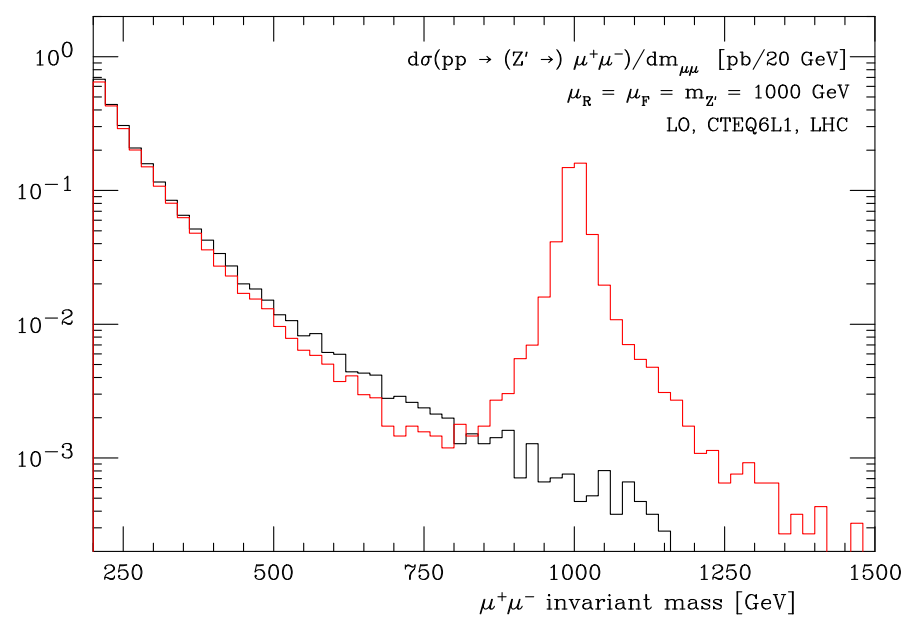

Figure 2: Dimuon invariant mass including a s-channel $Z^{\prime}$ resonance (red) and the SM spectrum (black). $M_{Z^{\prime}}=1 \mathrm{TeV}$, LHC, CTEQ6L1, no cuts.

As we have identified a new particle $X$, such as the $Z^{\prime}$, in the invariant mass distribution, the next step is be to study its properties. The most important quantum number is certainly its spin, which, in the dimuon channel, can be found by looking at the angular correlations between the muons. In this case, the angle $\theta$ introduced by Collins and Soper [12], can be used to access the spin information, minimizing at the same time the dependence on initial state radiation. The angle $\theta$ is defined as follows. Let $p_{A}$ and $p_{B}$ the momenta of the incoming hadrons in the rest frame of the muon pair. If the transverse momentum of the muon pair is non-zero, then $p_{A}$ and $p_{B}$ are not collinear. The angle $\theta$ is defined to be the angle between the axis that bisects the angle between $p_{A}$ and $p_{B}$ and the $\mu^{+}$momentum in the muon pair rest frame. In Fig. 3 the $\cos \theta$ is plotted for a spin- 0 , spin- 1 and a spin-2 particle $X$.

For the spin- 0 and spin- 1 particles the shapes of the $\cos \theta$ distributions are independent of their masses. This is not the case for the spin-2 particle. In contrast to spin- 0 particles, the spin of the spin-2 particle depends on the production mechanism and, contrary to spin-1 particles, this particle can be produced both by quark-anti-quark annihilation as well as gluon fusion. In Fig. 3 the distribution is plotted for a spin-2 particle with a mass of $1 \mathrm{TeV}$. 

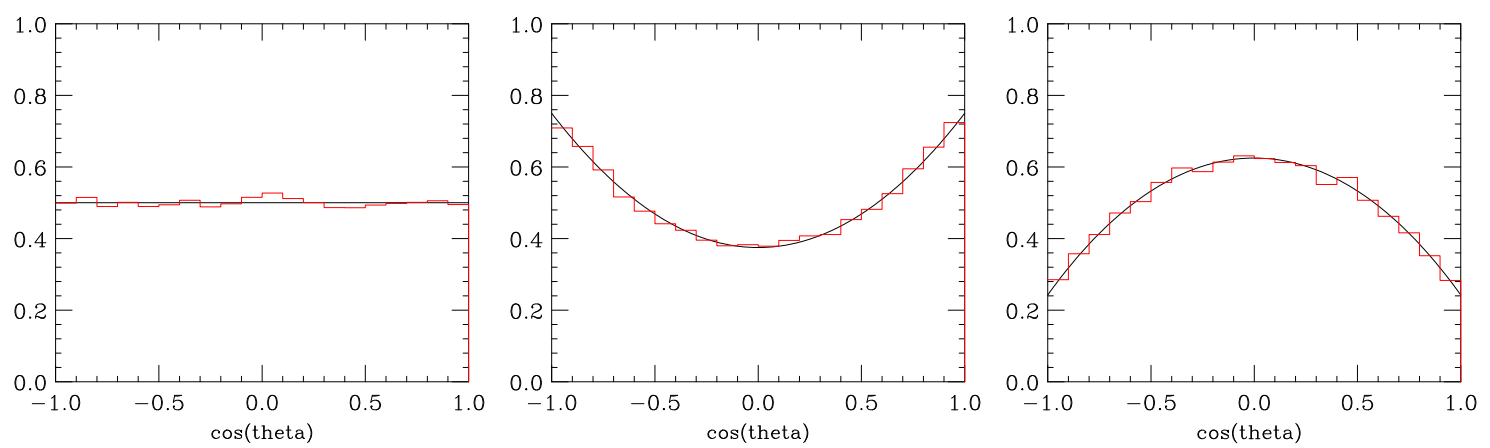

Figure 3: The normalized cross section as a function of $\cos \theta$ in $p p \rightarrow X \rightarrow \mu^{+} \mu^{-}$. Left for a spin- 0 particle, center for a spin-1 particle, right for a $1 \mathrm{TeV}$ spin-2 particle. No cuts applied.

\section{Heavy resonances in $t \bar{t}$}

If the coupling of the new particle $X$ to light particles is suppressed, e.g., in models with warped extra dimensions with bulk Standard Model fields, where the hierarchy is solved nonsuper-symmetrically [13], the dilepton channel could not be viable. In these models the resonances will have a large coupling to top quarks. The LHC, with a production of about $1 t \bar{t}$ pair every second, will be the first collider where this top quark invariant mass spectrum can be studied in detail up to several TeVs.

In the case where the width is not too large and the (possible) interference effects are not dominating, the resonance could be visible as a peak in the $t \bar{t}$ invariant mass distribution, just like the peak in the dimuon channel, see Section 2.2. Besides the experimental challenges coming from detecting/reconstructing the top quarks which we do not go into, there are also theoretical systematics that need to be addressed. Top production proceeds through an $\alpha_{S}^{2}$ processes, namely $g g \rightarrow t \bar{t}$ at the LHC, and there are potentially large uncertainties from higher order corrections which could affect areas of the phase space differently. In the next section we will discuss the theoretical errors in the $t \bar{t}$ invariant mass distribution.

\section{1 $t \bar{t}$ invariant mass spectrum}

In Fig. $\emptyset$ the SM $t \bar{t}$ invariant mass spectrum is plotted. This distribution is at next-to-leading order (NLO), calculated using MCFM [14], including the theoretical errors coming from the scale and PDF uncertainties. The (renormalization and factorization) scale uncertainties are estimated by varying the scales independently in the region between $\mu_{R}=\mu_{F}=m_{t} / 2$ and $\mu_{R}=\mu_{F}=2 m_{t}$ and the PDF uncertainties by running with the multiple 'error' PDF sets of the CTEQ6 family [15].

Running the 41 members of the CTEQ6 PDF-set gives a relatively small uncertainty of about $\pm 3.2 \%$ between the largest and the smallest values in the invariant mass spectrum. The total scale uncertainty at NLO is about $\pm 13 \%$. It is interesting to note that the scale uncertainties almost only change the normalization of the distribution, but not the shape. This means that this distribution is under good theoretical control; the normalization can be measured at any point of the distribution, which means that the scale uncertainties can become smaller in practice. Also, NLL resummed calculations indicate that the dependence on the scales could go down to $\pm 6 \%$ or even smaller [16], which suggests that the $\pm 13 \%$ estimated here, is very conservative as well. This, together 


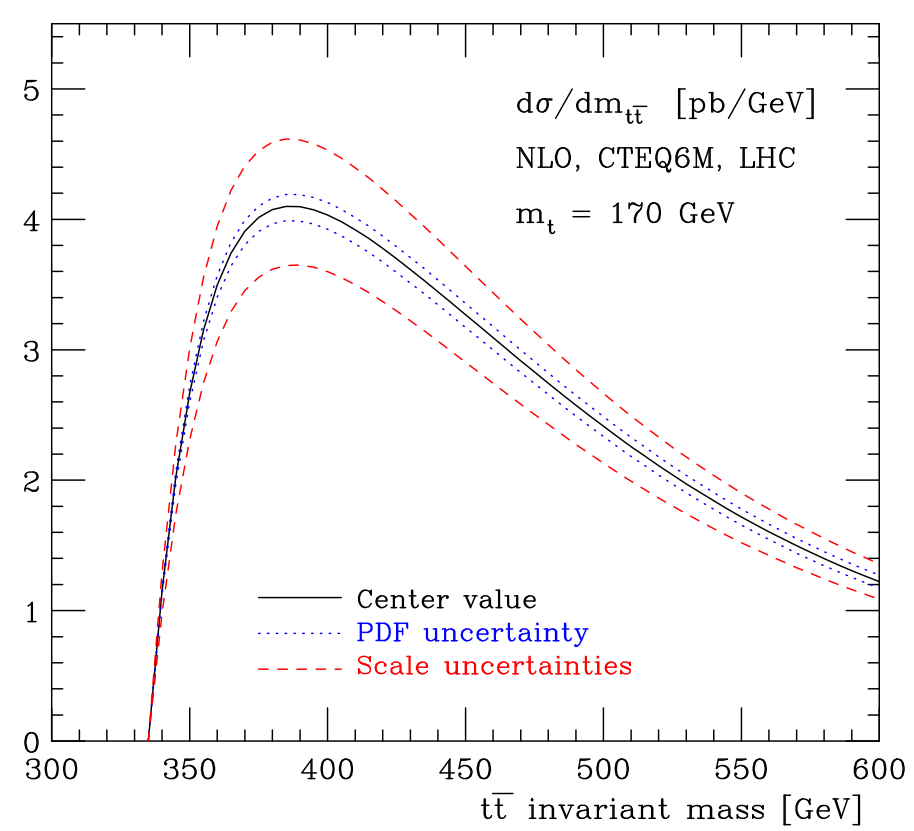

Figure 4: Scale (red) and PDF (blue) uncertainties in the $t \bar{t}$ invariant mass spectrum for $m_{t}=170 \mathrm{GeV}$, NLO, CTEQ6M, no cuts, complete mass reconstruction.

with the relatively clean leptonically decays of the top quarks, make the $t \bar{t}$ invariant mass spectrum a very promising distribution in the search for new physics.

On the other hand, experimental uncertainties coming from the reconstruction of the invariant mass spectrum and of the kinematics of the top quarks in the events, are going to be a real challenge [17].

\section{2 $X$ resonance}

In the previous section we showed that the $t \bar{t}$ invariant mass spectrum is under good theoretical control. Here we will see what the effect is of a $X$ resonance on the $t \bar{t}$ invariant mass spectrum. Including an $s$-channel resonance in the $t \bar{t}$ production process produces a clear peak in the invariant mass spectrum as can be seen in Fig. 5. The precise width and height of the peak depends on the model parameters. As a benchmark we show a $Z^{\prime}$ vector boson with mass $m_{Z^{\prime}}=600 \mathrm{GeV}$ that couples with the same strength to quarks as a Standard Model $Z$ boson.

At the theoretical level, the resonance is very clearly visible in the $t \bar{t}$ invariant mass spectrum and its observability at the LHC will depend only on the experimental resolution on $m_{t \bar{t}}$. If a resonance is found, the next step will be to study its properties and quantum numbers. Again we would like to identify the spin of the resonance.

\subsection{Spin correlations}

Due to the decay of the top quarks, the study of the spin of the resonance is consideredly more difficult compared to the dilepton channel, Section 2.2. The spin structure can studied by looking at the angular correlations between the decay products of the top quarks. The double leptonic decay, i.e., both the top and the anti-top decay leptonically $t \rightarrow b W \rightarrow b l v$, see Fig. 6 , is the best channel 


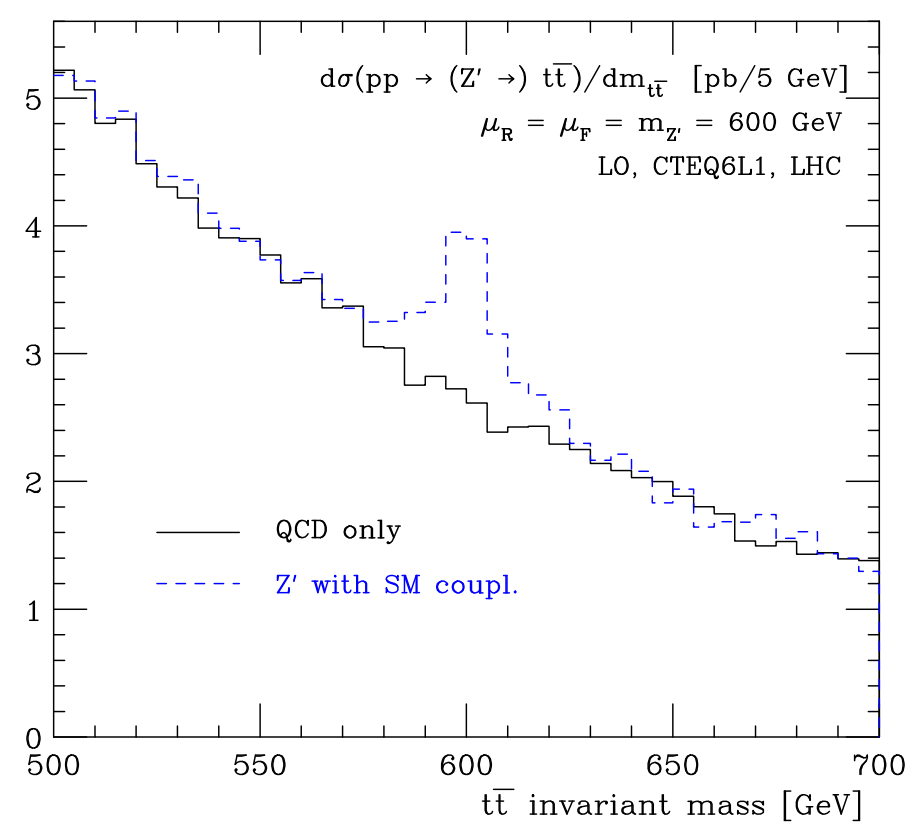

Figure 5: Invariant $t \bar{t}$ spectrum for $p p \rightarrow t \bar{t}$ including a $s$-channel $Z^{\prime}$ vector boson with mass $m_{Z^{\prime}}=600 \mathrm{GeV}$ that couples with Standard Model strength to quarks. Black without the $Z^{\prime}$, blue with the $Z^{\prime}$. CTEQ6L1, $\mu_{R}=\mu_{F}=600 \mathrm{GeV}$, no cuts.

to search for the spin correlations because of the very clear leptons. The angular distributions of the two leptons gives us information about the spin of the (anti-)top quarks.

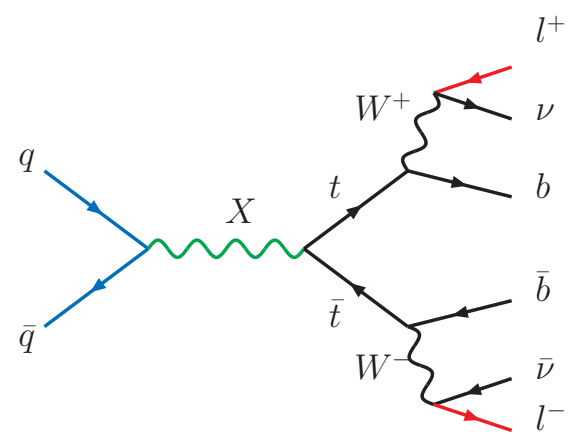

Figure 6: One representative Feynman diagram contributing to $p p \rightarrow X \rightarrow t \bar{t}$, with double leptonic top quark decay.

Supposing that a beyond the SM resonance is found through a sharp peak, we can look only at the effects of the resonance on the spin correlations, not taking into account the background QCD $t \bar{t}$ production.

The following double distribution is usually considered in studies on the spin correlations in $t \bar{t}$ production, see e.g., Ref. [18],

$$
\frac{1}{\sigma} \frac{d^{2} \sigma}{d \cos \theta_{+} d \cos \theta_{-}}
$$


where $\theta_{+}\left(\theta_{-}\right)$is the angle between the $t(\bar{t})$ direction in the $t \bar{t}$ center of momentum frame and the $l^{+}\left(l^{-}\right)$direction in the $t(\bar{t})$ rest frame. To reconstruct the top momenta, the momenta of the undetected neutrinos are needed. Imposing kinematic constraints, such as the known top and W masses, a system of equations for the neutrino momenta can be set-up. Although in general multiple solutions arise weights can be given to each of them to obtain the best solution for the momenta [18]. For the sake of illustration we assume that the top quark momenta are correctly reconstructed.

In Fig. 7 the double distribution (3.1) is plotted for QCD $t \bar{t}$ production.

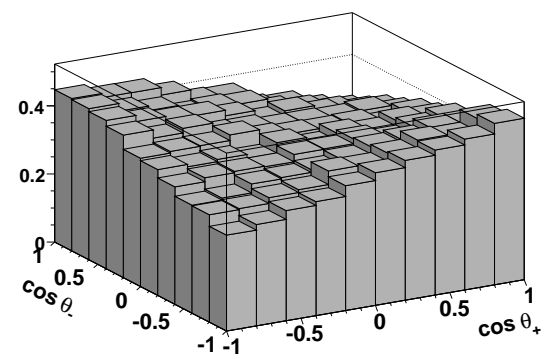

Figure 7: The distribution $d^{2} \sigma / d \cos \theta_{+} d \cos \theta_{-}$for SM $t \bar{t}$ production at the LHC, CTEQ6L1, no cuts.

There are clear differences in this distribution for the various $t \bar{t}$ production mechanisms. In Fig. 8 the distributions are plotted for resonance masses of $800 \mathrm{GeV}$. A scalar boson, vector boson and spin-2 boson resonances are shown. We choose very narrow resonances by taking the width of resonances to be $1 \%$ of the mass, i.e., $8 \mathrm{GeV}$ for the resonances of $800 \mathrm{GeV}$. We neglect the SM $\mathrm{QCD} t \bar{t}$ production background in these plots.

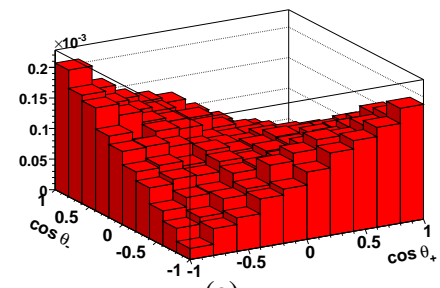

(a)

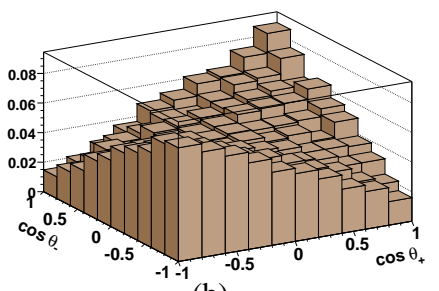

(b)

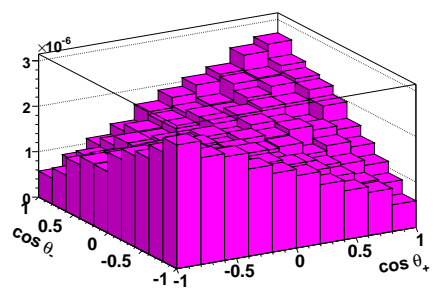

(c)

Figure 8: The distribution $d^{2} \sigma / d \cos \theta_{+} d \cos \theta_{-}$for (a) scalar, (b) vector, (c) spin-2. $M_{X}=800 \mathrm{GeV}, \mathrm{LHC}$, CTEQ6L1, no cuts.

The three double distributions are clearly different from the SM distribution. But the distributions for the vector and the spin-2 resonances are very similar and other methods should be developed and used to distinguish between these two cases.

\subsection{Interference effects in a pseudo-scalar resonance}

So far we have considered the case where new particles do not interfere with the SM $t \bar{t}$ production. Besides these "simple resonances" there are possibly other effects that BSM physics might entail on the $t \bar{t}$ invariant mass spectrum. As a non trivial example, let us consider a pseudo-scalar Higgs $A$ resonance. For a (pseudo-)scalar resonance the signal amplitude, i.e., gluon fusion through a quark loop, interferes with the background QCD $t \bar{t}$ production, see Fig. $\vartheta$. 

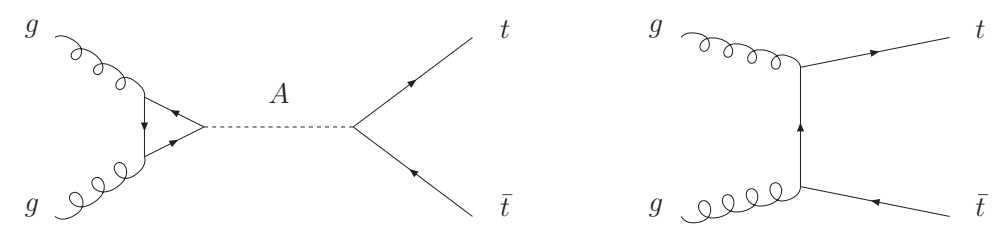

Figure 9: Feynman diagram for the $A$ resonance (left), and one of the SM $t \bar{t}$ production diagrams with which it interferes (right).

In a minimal extension of the Standard Model where the pseudo-scalar boson coupling strength to quarks, is proportional to the mass of the quarks $m_{q}$, we have

$$
g_{A q q}=a_{2} \frac{m_{q}}{v} \gamma_{5}
$$

Here, $v$ is the Higgs field vacuum expectation value and $a_{2}$ is a dimensionless constant that measures the size of the coupling. Because the top quark is by far the most massive quark, its contribution dominates in the loop. If the mass of the Higgs boson is larger than twice the mass of the top quark, the loop-induced gluon-gluon-Higgs coupling gets an imaginary part, which leads to a peak-dip structure for the interference terms between the QCD background and the Higgs signal [19, 20].

At tree-level, a pseudo-scalar decays only to fermions, if we can neglect decays to the $Z h$, where $h$ is a scalar Higgs boson. And, because the coupling of the pseudo-scalar to quarks is proportional to the fermion mass, we assume that it decays only to $t \bar{t}$ pairs, i.e., $\operatorname{BR}(A \rightarrow t \bar{t})=1$. The interference between the signal and the QCD $t \bar{t}$ production leads to a dip in $t \bar{t}$ production at an invariant mass just above the Higgs boson mass. The signal together with the interference terms sum then to the characteristic 'peak-dip' structure, shown in Fig. 10. The red line in Fig. 10 shows the effect of a $400 \mathrm{GeV}$ pseudo-scalar Higgs boson on the $t \bar{t}$ invariant mass spectrum with coupling constant $a_{2}=1$. Comparing with the QCD $t \bar{t}$ production, the black line, a clear peak-dip structure is visible. If the coupling to top quarks is enhanced, the peak as well as the dip get broader due to the larger decay width. The green line shows the effects of a coupling twice as large as the red line. If the coupling to the top quarks gets even larger, the increasing width of the (pseudo-)scalar starts to dominate, the dip disappears and only a broad peak remains.

If, on the contrary, the coupling to the top is smaller than unity, the peak and the dip become narrower. For $a_{2}=0.5$, the blue line in Fig. 10, a very clear peak-dip structure is still visible, although it becomes more difficult to resolve.

\section{Conclusions}

In this talk I presented the MadGraph/MadEvent MC event generator by discussing a simple new physics scenario, i.e., the discovery and the study of a new s-channel resonance in $p p$ collisions at the LHC. To perform the analyses shown here, we have run over the MG/ME clusters at

http: //madgraph.phys.ucl.ac.be/

http://madgraph.hep.uiuc.edu/ 


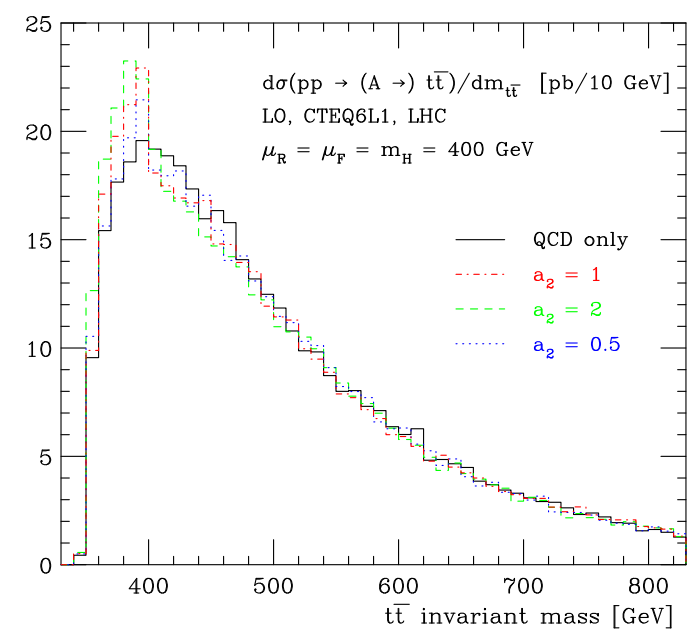

(a)

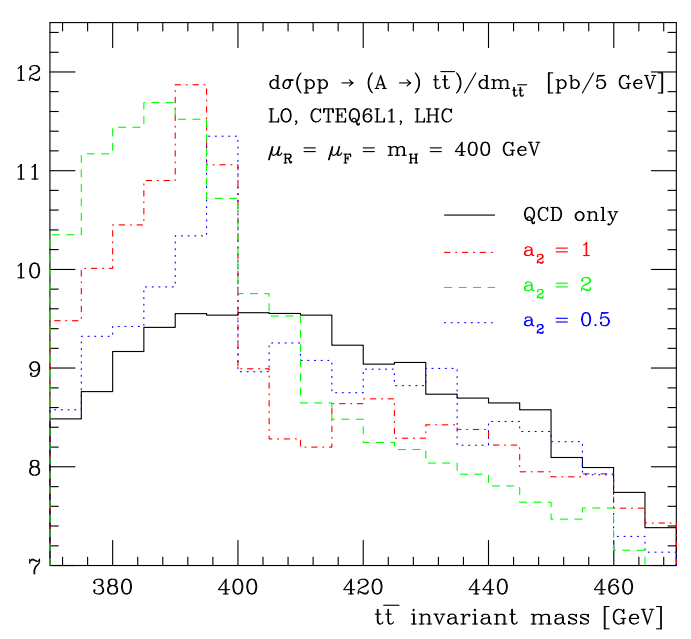

(b)

Figure 10: Invariant $t \bar{t}$ mass spectrum for the pseudo-scalar Higgs. Right: The interesting region with finer binning. Different colors represent different coupling strength of the Higgs to top quarks: Red for the standard model coupling and blue and green for 0.5 and 2 times the standard model coupling strength, respectively. Black is QCD $t \bar{t}$ production, i.e. without the Higgs signal. CTEQ6L1, $\mu_{R}=\mu_{F}=400 \mathrm{GeV}$, no cuts.

http://madgraph.roma2.infn.it/

where anybody can access and perform similar studies.

\section{Acknowledgments}

I would like to thank Fabio Maltoni for all the useful discussions and comments, Simon de Visscher for developing the user model and also all the other colleagues in the MadGraph/MadEvent team: Johan Alwall, Pavel Demin, Michel Herquet, Tilman Plehn, David Rainwater and Tim Stelzer.

\section{References}

[1] T. Stelzer and W. F. Long, Automatic generation of tree level helicity amplitudes, Comput. Phys. Commun. 81 (1994) 357-371, hep-ph/9401258].

[2] F. Maltoni and T. Stelzer, MadEvent: Automatic event generation with MadGraph, JHEP 02 (2003) 027, hep-ph/0208156].

[3] J. Alwall et. al., MadGraph/MadEvent v4: The New Web Generation, 0706.2334.

[4] H. Murayama, I. Watanabe, and K. Hagiwara, HELAS: HELicity Amplitude Subroutines for Feynman diagram evaluations, KEK-91-11.

[5] T. Sjostrand, S. Mrenna, and P. Skands, PYTHIA 6.4 physics and manual, JHEP 05 (2006) 026, hep-ph/0603175].

[6] G. Corcella et. al., HERWIG 6: An event generator for hadron emission reactions with interfering gluons (including supersymmetric processes), JHEP 01 (2001) 010, hep-ph/0011363. 
[7] J. Conway et al., PGS 4: Pretty Good Simulation of high energy collisions, http://www.physics.ucdavis.edu/ conway/research/software/pgs/pgs4-general.htm.

[8] G.-C. Cho et.al., Weak boson fusion production of supersymmetric particles at the LHC, Phys. Rev. D73 (2006) 54002, [hep-ph/0601063].

[9] T. Appelquist, H. Cheng and B. Dobrescu, Bounds on universal extra dimensions, Phys. Rev. D64 (2001) 35002, [hep-ph/0012100].

[10] P. Skands et. al., SUSY Les Houches accord: Interfacing SUSY spectrum calculators, decay packages, and event generators, JHEP 07 (2004) 036, hep-ph/0311123.

[11] P. Meade and M. Reece, BRIDGE: Branching ratio inquiry / decay generated events, [hep-ph/0703031].

[12] J. C. Collins and D. E. Soper, Angular Distribution of Dileptons in High-Energy Hadron Collisions, Phys. Rev. D16 (1977) 2219.

[13] R. Contino, T. Kramer, M. Son, and R. Sundrum, Warped/Composite Phenomenology Simplified, [hep-ph/0612180].

[14] J. M. Campbell and R. K. Ellis, An update on vector boson pair production at hadron colliders, Phys. Rev. D60 (1999) 113006, hep-ph/9905386].

[15] J. Pumplin et. al., New generation of parton distributions with uncertainties from global QCD analysis, JHEP 07 (2002) 012, hep-ph/0201195].

[16] R. Bonciani, S. Catani, M. L. Mangano, and P. Nason, NLL resummation of the heavy-quark hadroproduction cross- section, Nucl. Phys. B529 (1998) 424-450, [hep-ph/9801375].

[17] V. Barger, T. Han, and D. G. E. Walker, Top quark pairs at high invariant mass: A model-independent discriminator of new physics at the LHC, hep-ph/0612016.

[18] M. Beneke et. al., Top quark physics, hep-ph/0003033].

[19] K. J. F. Gaemers and F. Hoogeveen, Higgs production and decay into heavy flavors with the gluon fusion mechanism, Phys. Lett. B146 (1984) 347.

[20] D. Dicus, A. Stange, and S. Willenbrock, Higgs decay to top quarks at hadron colliders, Phys. Lett. B333 (1994) 126-131, [hep-ph/9404359]. 